\title{
Determination of thermal and acoustic comfort inside a vehicle's cabin
}

\author{
Alexandra Ene (Angelescu) $)^{1,}$, Tiberiu Catalina ${ }^{1 *}$, and Andreea Vartires $^{1}$ \\ ${ }^{1}$ Technical University of Civil Engineering, Faculty of Building Services, Romania
}

\begin{abstract}
Thermal and acoustic comfort, inside a vehicle's cabin, are highly interconnected and can greatly influence the health of the passengers. On one hand, the H.V.A.C. system brings the interior air parameters to a comfortable value while on the other hand, it is the main source of noise. It is an intriguing task to find a balance between the two. In this paper, several types of air diffusers were used in order to optimize the ratio between thermal and acoustic interior comfort. Using complex measurements of noise and thermal comfort parameters we have determined for each type of air diffuser the sound pressure level and its impact on air temperature and air velocity.
\end{abstract}

\section{Introduction}

Nowadays, many people spend a significant proportion of their time travelling and there is an increasing demand for comfort, in both private and public transport [1].

Noise and vibrations have an important influence on a customer's perception of vehicle quality and interior cabin noise levels are key criteria when choosing a vehicle [2]. Further on, the HVAC system represents one of the most important parameters that influences the interior comfort of a vehicle's cabin. On one hand it represents necessary equipment in order to obtain thermal comfort while on the other hand, it represents the most important parameter that influences negatively the acoustic comfort, due to air flows from the multitude of the HVAC settings. Different supply air velocity and temperature on the car chamber has a great influence on air distribution characteristics [3].

Commonly, vehicle occupants choose to operate the vehicle ventilation system in the "recycle" mode to prevent outdoor-polluted air entering the vehicle's cabin and by that raising the concentration of $\mathrm{CO}_{2}$ [4].

The thermal comfort sensation is assured by the factors that depend on the heat exchange between the human body and the ambient environment [5].

The ventilation and air conditioning system consists of a fan, a mixing unit (formed from various equipment) and air ducts that lead to the places where the air enters the cabin, including the front grids, the grids from the lower/feet area, for both the front and back passengers, demisting and defrosting actions, and other combinations depending on the type and construction of the chosen car [6].

\section{Experimental campaign}

\subsection{Acoustic measurements}

The determinations were performed on a 2012 Dacia Duster vehicle, in an enclosed room in order to maintain external background noise as low as possible. The tested vehicle presents different positions through which air is introduced into the cabin, as shown in Fig. 1. The grids numbered with " 1 " have a demisting role. The four ones, numbered with " 2 ", introduce the air towards the face of the occupants and the ones numbered with " 3 " can introduce air at the bottom part of the cabin where the passenger's feet should be.

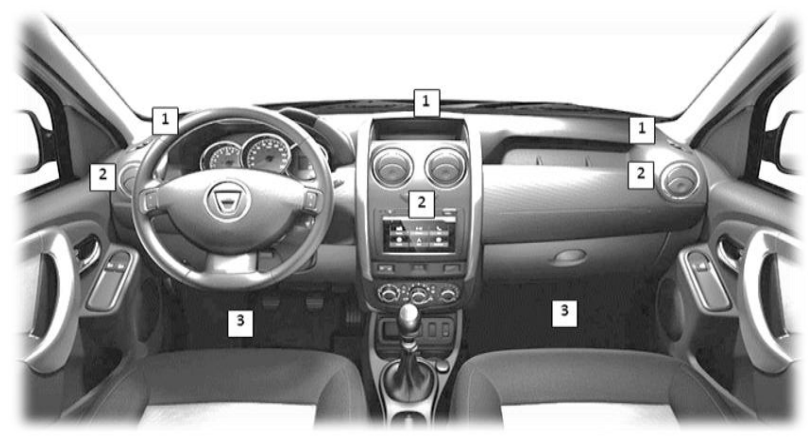

Fig. 1. Dacia Duster vehicle's interior 
The fan presents four different gears through which the airflow introduced into the cabin can be changed, as shown in Fig. 2. The car also presents five types of combinations which can be chosen for introducing the air into the cabin, formed from a combination of the three positions shown in Fig. 1.

-Position (a): the air is introduced for demisting (fig.1, grids “1”);

-Position (b): the air is introduced for demisting and the lower/feet area (fig. 1, grids " 1 " and " 3 ");

-Position (c): the air is introduced for the lower/feet area (fig. 1, grids " 3 ");

-Position (d): the air is introduced towards the face and for the lower/feet area (fig.1, grids " 1 " and "3");

-Position (e): full towards the face (fig.1, grids " 2 ").

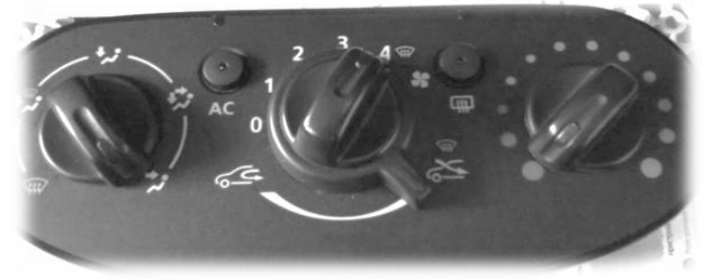

Fig. 2. Methods for adjusting the H.V.A.C. system

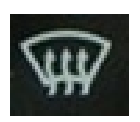

(a)

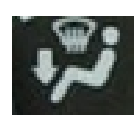

(b)

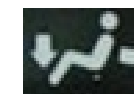

(c)

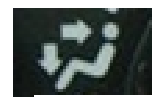

(d)

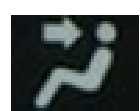

(e)
Fig. 3. Typical vehicle HVAC system operations

\subsubsection{Equipment used in the study}

The sound pressure levels were determined by means of a sound level meter Bruel\&Kjaer 3560-B-020, precision class 1 . The sonometer presents five receivers giving the ability to measure noise level from five different points, simultaneously.

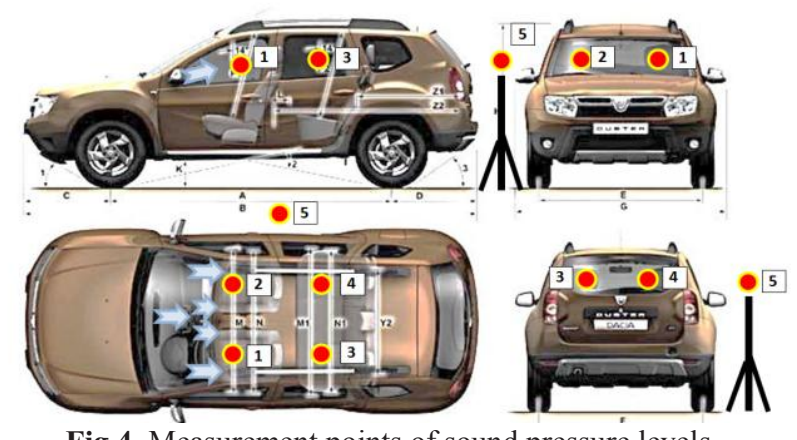

Fig.4. Measurement points of sound pressure levels

The receivers were placed on each of the points occupied by a passenger, as shown in Fig. 4. Point "1" represents the driver's position, point " 2 " the front passenger, near to the driver's seat, point " 3 " the passenger from the back of the chauffeur and the "4" point corresponds to the back passenger who seats diagonally from the driver's chair. The exterior sound pressure level was constantly measured by receiver " 5 " placed outside the car at a distance of about $0,5 \mathrm{~m}$.

The experimental campaign was conducted for five different air vents. The first one used was the initial air diffuser of the car, the second one - air diffuser spherical type one (prototype 1), third one - air diffuser spherical type two (prototype 2), fourth - air diffuser propeller type (prototype 3), fifth - classic air diffuser type (prototype 4).

First of all, the sound pressure level with the ventilation system off was measured, then the measurements were conducted for every fan gear, for every position of air inlets and also replacing the originals air grids with the four new prototypes. The sound pressure level was recorded for 20 seconds in each mentioned case.

The sound pressure level resulted exclusively from the ventilation system as a difference between the sound pressure level measured with the ventilation system on (for the mentioned cases) and the sound pressure level measured with the ventilation system off.

The results were downloaded on the computer by means of the software "Labshop" and the data was imported into an Excel document.

The air flows for every fan gear were determined by placing the Testo 480's anemometer at the air inlet. The air flows resulted for every fan gear are: Gear $1=169.5$ $\mathrm{m}^{3} / \mathrm{h}$, Gear 2=251.0 $\mathrm{m}^{3} / \mathrm{h}$, Gear $3=343.2 \mathrm{~m}^{3} / \mathrm{h}$, Gear $4=451.3 \mathrm{~m}^{3} / \mathrm{h}$.

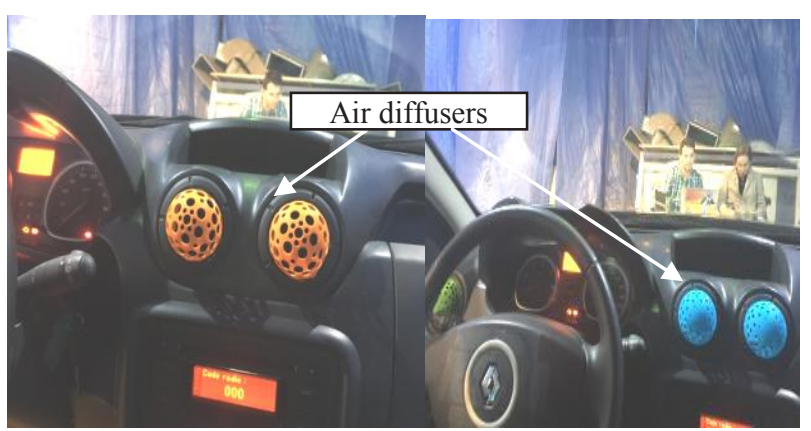

Fig.5. Photographs taken while measurement campaign was going

\subsubsection{Results of the acoustic experimental campaign}

The results display the fact that by using the demisting air vents, the interior sound pressure level is highest, up to $5 \mathrm{dBA}$ higher than the others, representing a value which can be detected by the human ear. The air vents present a higher contribution on the sound pressure level because of the air hitting solid surfaces. Furthermore, air entering the cabin in full face mode represents the second highest sound pressure level. The sound pressure level differences between air vents is overall small, the highest one being of $2 \mathrm{dBA}$. 


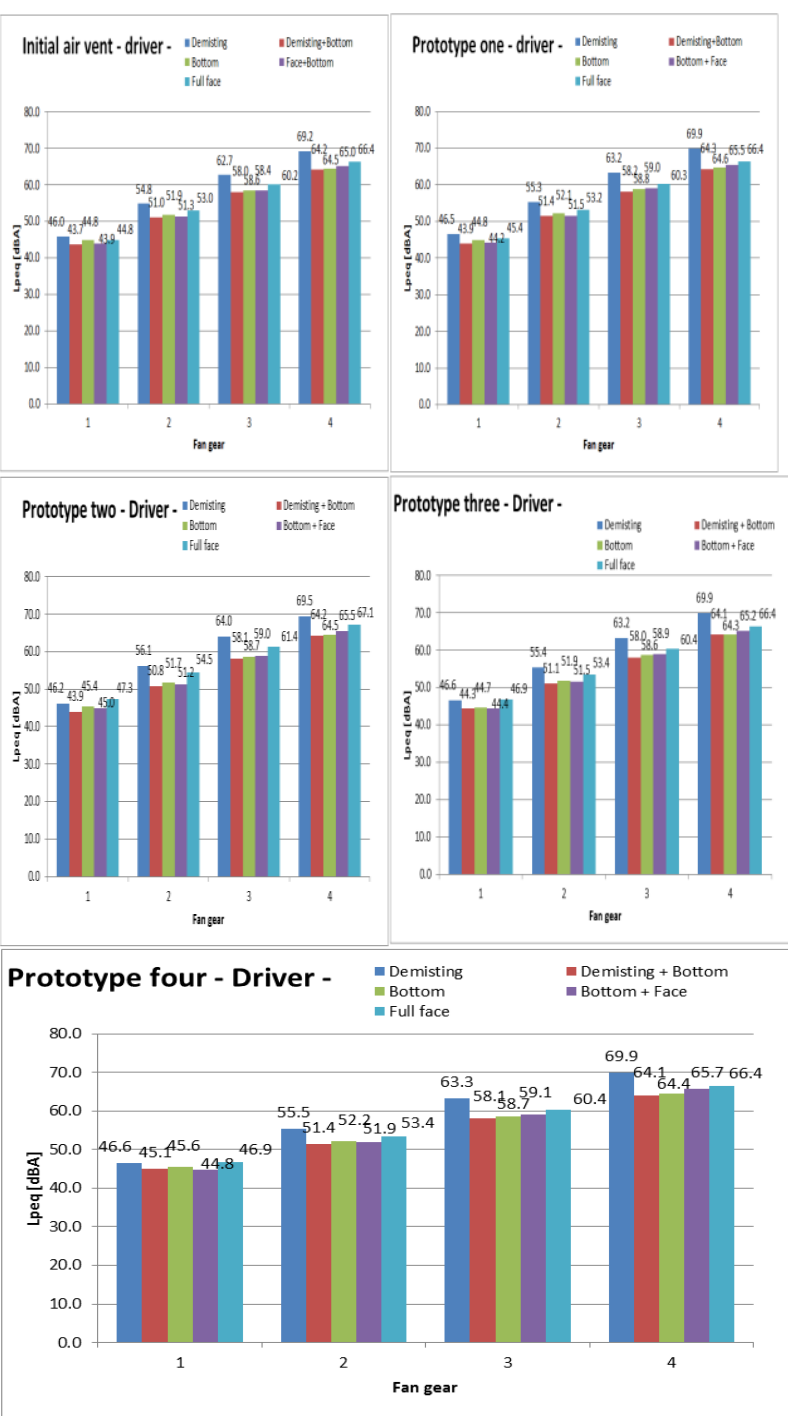

Fig. 6. Sound pressure level for driver position with different air vents

The results showed also a rise of $48.2 \%$ of the sound pressure level for gear 4 of the fan, comparing with a rise of the air flow of $166 \%$ from gear 1 of the fan. Further on, the graphics show a difference up to $5 \mathrm{dBA}$ sound pressure level between the front and the back passengers' positions for every type of air vent analyzed.

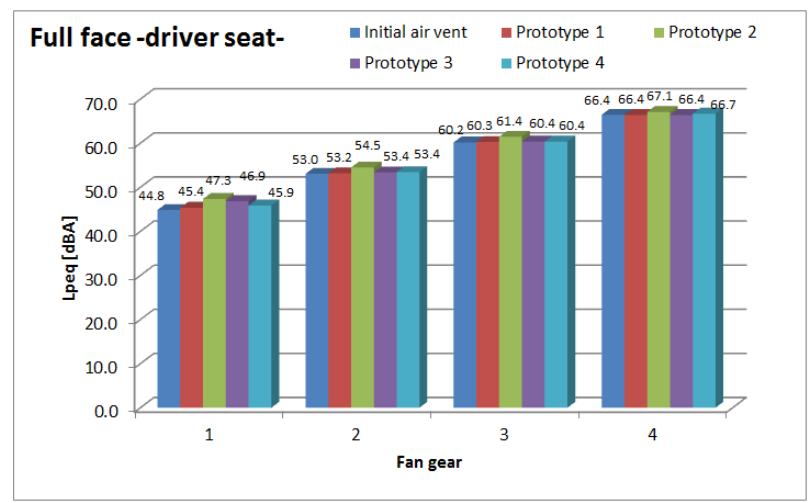

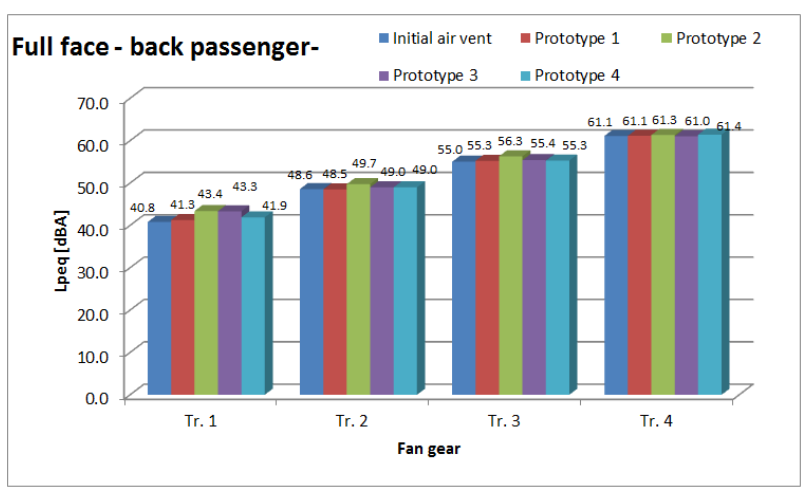

Fig. 7. Sound pressure level for driver and back passenger's position with different air vents

\subsection{Thermal comfort measurements}

The ventilation and air conditioning system presents the role of maintaining the interior air parameters in comfort conditions for passengers. The main factors which affect the human thermal comfort depend on four physical aspects which vary with the environment:

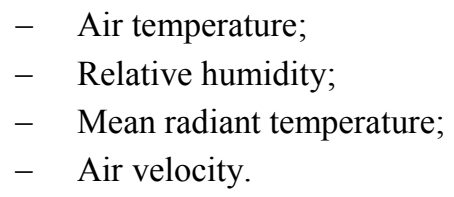

The interior environment of a vehicle is affected by a large number of parameters in which can be included the indoor and outdoor temperature, solar radiation, geometrical characteristics and materials, reflection coefficient and also thermal mass of the interior materials. All these factors slow the calculation method and the predictability of the thermal comfort. For this reason in the present paper, the analyzed situation is the one in which a part of these factors were eliminated with the purpose of a more accurate determination of the interior comfort, without the external intervention of individuals presenting factors which are difficult to predict.

This measurement campaign was conducted in an indoor room with the purpose of eliminating the direct solar radiation. Also, the same model of vehicle, presented before, was used.

\subsubsection{Equipment used in the study}

For this experimental campaign, two identical equipment Testo 480 were used, which contain multiple devices for measuring the IAQ, for calculation and analyzing PMV/PPD, air temperature, air velocity etc. 


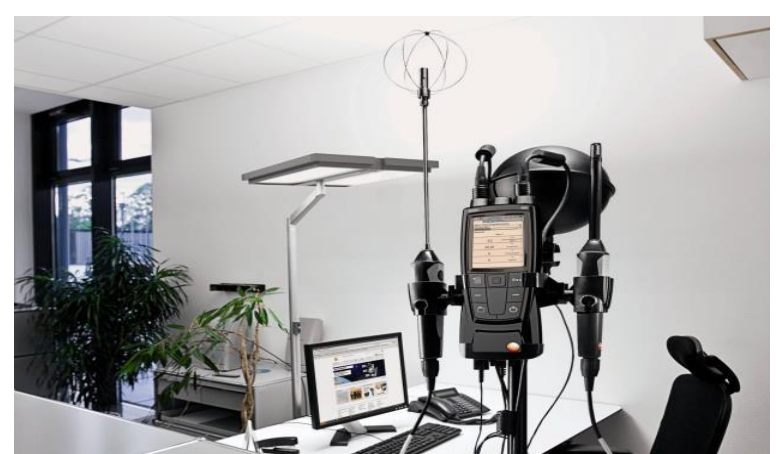

Fig. 8. Equipment used for the comfort determinations Testo 480

The determinations were conducted in an enclosed space in order to eliminate various factors like direct solar radiation. The two Testo 480 equipment were placed in the car (one on the driver's seat, and the second one on the back passenger's seat) as shown in fig. 9 .

The HVAC system was set in order to introduce fresh air from the outside, with the recycling mode off, fan gear two and the air conditioning on. Every determination lasted ten minutes and consisted in 600 measurements. For every new phase, it was needed to get inside the vehicle and save the data. Also in order to determine the PMV and PPD indices, cloth isolation was set at 0.6 clo and the metabolism 1 met. During the experimental campaign the air temperature raised from $30^{\circ} \mathrm{C}$ to $34^{\circ} \mathrm{C}$.
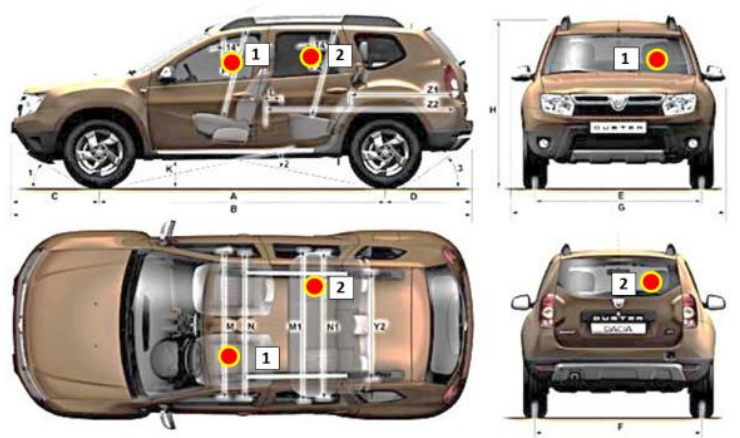

Fig. 9. Measurement points for the comfort analysis

\subsubsection{Results of the thermal comfort experimental campaign}

The results showed a significant air temperature difference between the front and the back passengers' position. This difference can lead up to $2^{\circ} \mathrm{C}$ when using the full face inlets and the combination of face and lower air grids. Furthermore, the indoor air temperature raises from about $23{ }^{\circ} \mathrm{C}$ (for the air diffusers propeller type) to $30{ }^{\circ} \mathrm{C}$ at the end of the measurement campaign (for the car's initial air diffusers).

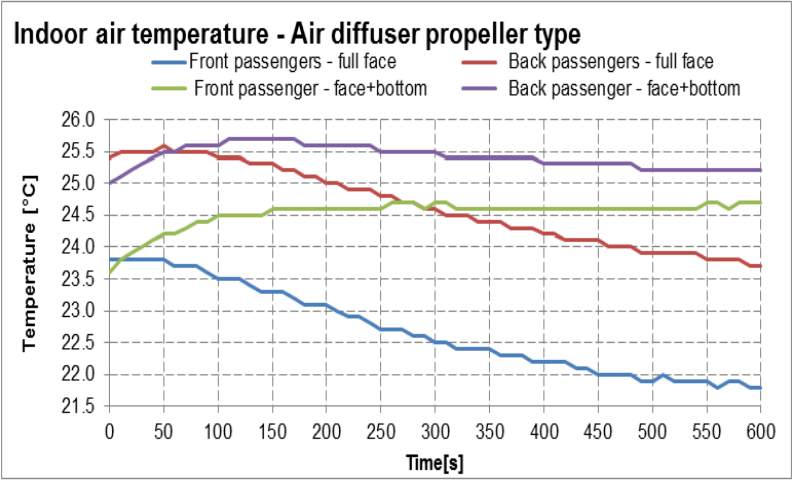

Indoor air temperature - Initial air diffusers' car

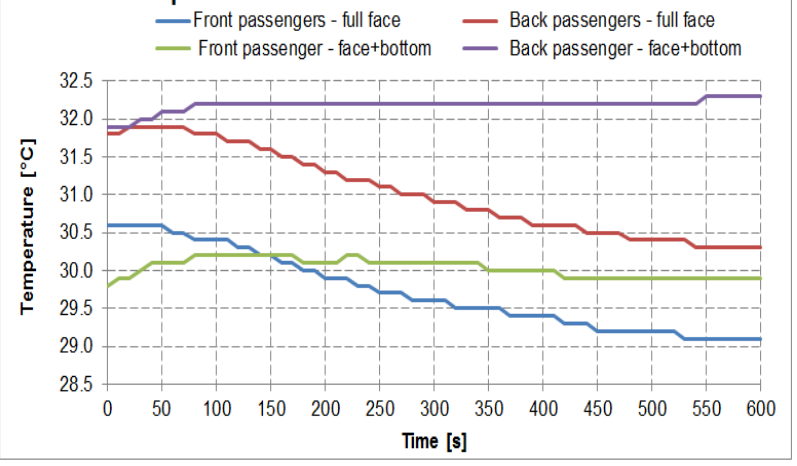

Fig. 10. Variation of the indoor air temperature during measurement campaign

\subsubsection{Results of the second thermal comfort experimental campaign}

Due to the fact that indoor air temperature rose drastically through the experimental campaign, it was decided to retake the measurements. Position and time of the measurement remained the same as in the first campaign, the only difference being that the car was placed outdoor, screened away from the direct solar radiation. However, the outdoor air temperature raised 2 ${ }^{\circ} \mathrm{C}$ while the measurement campaign took place.

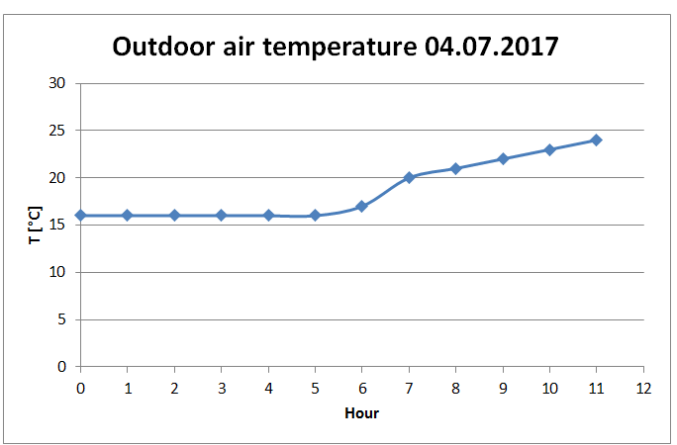

Fig. 11. The evolution of the outdoor air temperature in the day of the measurement campaign

Further on, the air conditioning system was turned off due to cold temperatures outside. 


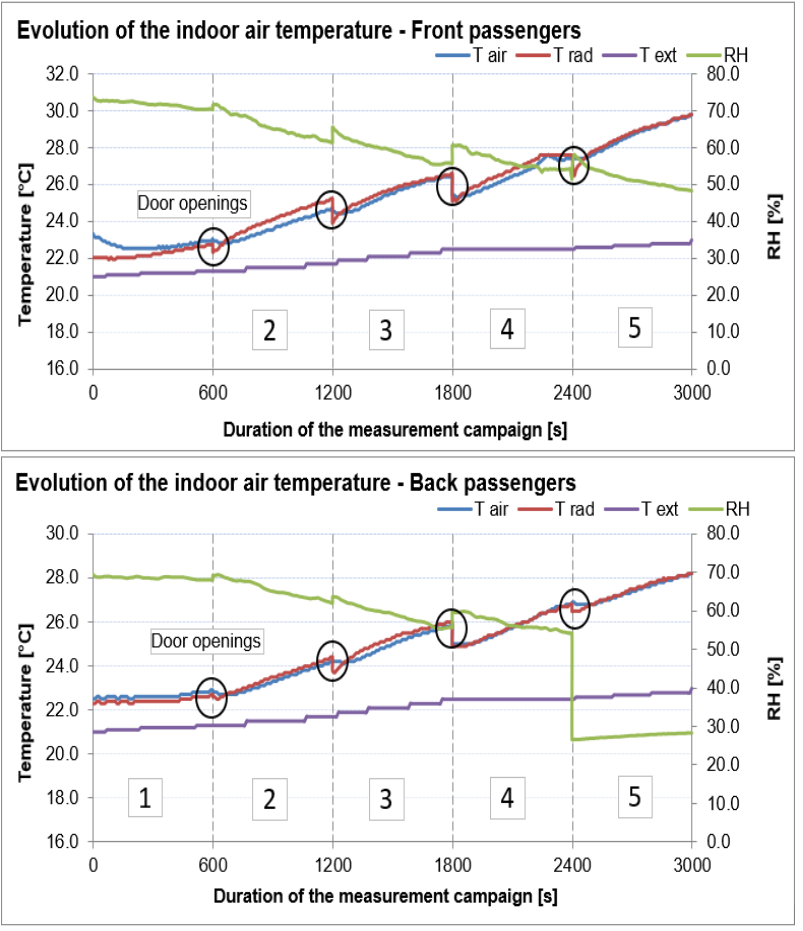

Fig. 12. The evolution of the indoor air temperature

As Fig. 12 shows, the indoor air temperature raises drastically during the measurement campaign, while the outdoor temperature remains constant. The vertical lines mark the measurement sets. If at the beginning of the measurement, indoor air temperature was about $22.5^{\circ} \mathrm{C}$ and the outdoor air temperature $21^{\circ} \mathrm{C}$, at the end of the campaign the interior air temperature in the cabin's vehicle reaches $29.9^{\circ} \mathrm{C}$ even though the outside air temperature doesn't exceed $23^{\circ} \mathrm{C}$.

Likewise, the door openings were highlighted with a circle. Regarding the back side of the car, the air temperature differences can still be identified.

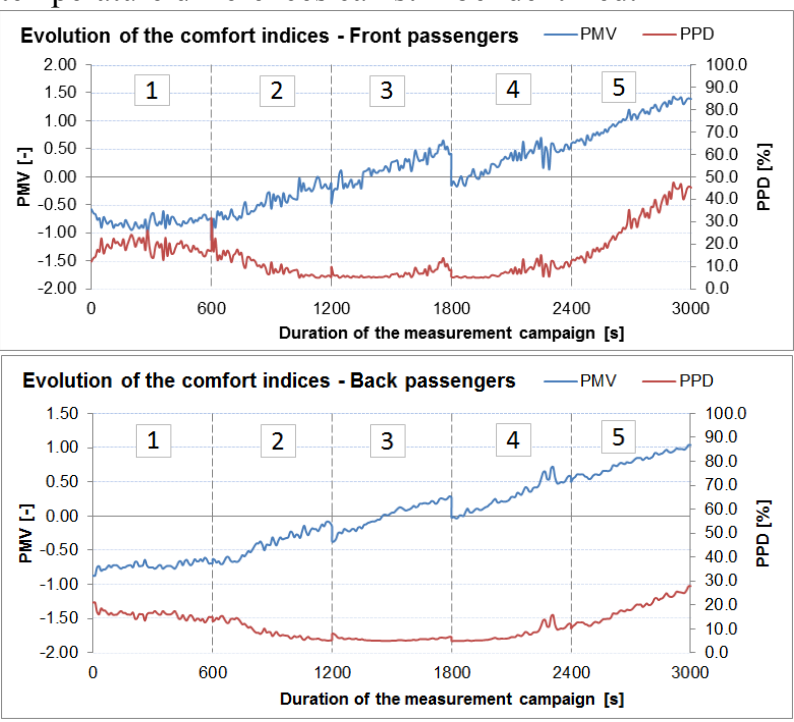

Fig. 13. The evolution of the PMV and PPD indices

Simultaneously with the raise of the air temperature the PMV and PPD indices are progressing, thus, if at the beginning of the measurement campaign PMV has the value of -0.5 , at the end of the measurements the value does not exceed 1 .
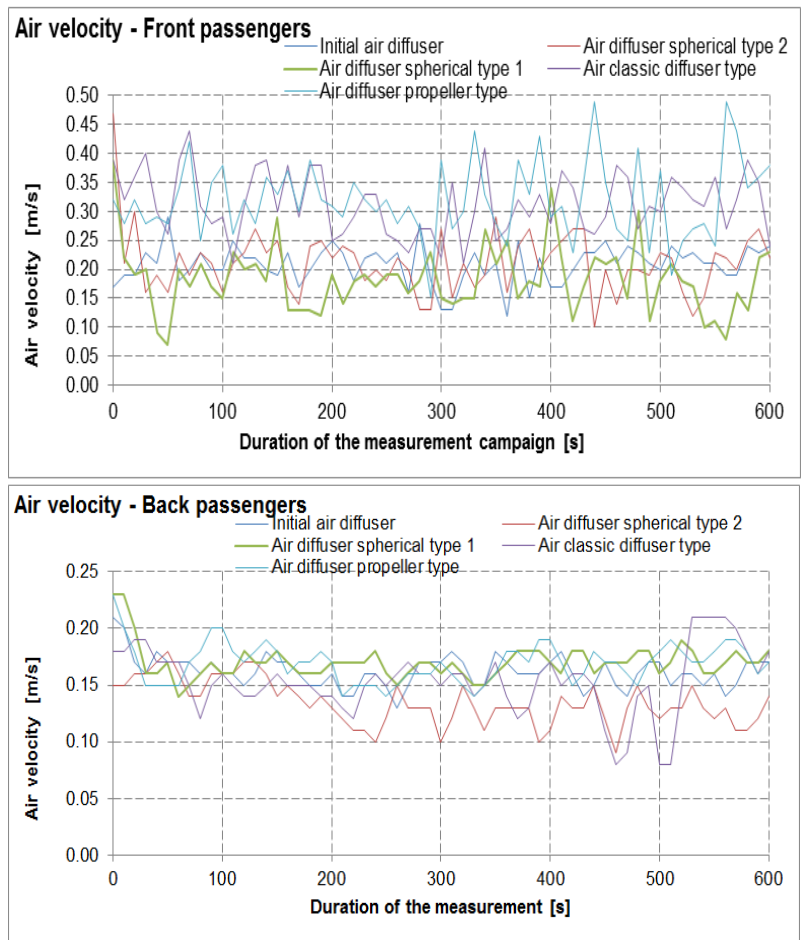

Fig. 14. Air velocity introduced by different air vents

Due to the fact that the temperature inside the vehicle varies excessively, the only way to compare the air vents is through air velocity. As it can be seen in Fig. 14, the initial air vents of the car can introduce an air velocity up to $0.45 \mathrm{~m} / \mathrm{s}$, representing the highest values. Analyzing the average values of the air velocity, it can be concluded that air diffusers spherical type 1 determine the lowest value at about $0.183 \mathrm{~m} / \mathrm{s}$. Regarding the back passengers, they would have preferred the use of the air diffuser spherical type 2 which can introduce a decrease down to $17 \%$, unlike the initial air diffuser of the car.

\section{Conclusion}

For the acoustic comfort it can be concluded that:

- The sound pressure levels coming from the HVAC system are higher than the ones coming from the engine;

- The acoustic differences between air vents are very low;

- In order to reduce the noise level, a damper is needed.

As for the thermal comfort inside the vehicle's cabin, it can be concluded that:

- The air temperature inside the cabin is higher than the outside air temperature;

- The air diffusers spherical type introduce the lowest values for air velocity;

- The air temperature inside the cabin increases simultaneously with the raise of the operating time of the vehicle, giving the need of using the air conditioning even though the outside temperature is at a comfortable value; 
- The vehicle presents a difference of $2{ }^{\circ} \mathrm{C}$ between front and back passengers.

\section{Acknowledgements}

This work was supported by a grant of the Romanian National Authority for Scientific Research, UEFISCDI, project number PN-II- PT- PCCA-2013- 4-0569.

\section{References}

1. Gameiro da Silva M. C., Measurements of comfort in vehicles, Measurement Science and Technology, (2002)

2. Watkins S., Wang X., Mavuri S. P., An Investigation and Bench Marking of Vehicle HVAC Cabin Noise, SAE TECHNICAL PAPER SERIES (2008)

3. Yang H. s.a., The Analysis on the Effect of Passenger car Air Conditioning and Distribution with Different Inlet Parameters, International Conference on Information Sciences, Machinery, Materials and Energy, (2015)

4. Wells B., McDonald S.,Vehicle Cabin Air Quality Monitor for Fatigue and Suicide Prevention, SAE TECHNICAL PAPER SERIES, (2000)

5. Simion M. s.a., Factors which influence the thermal comfort inside of vehicles, Sustainable Solutions for Energy and Environment, EENVIRO - YRC 2015, (2015)

6. Angelescu A. s.a., Acoustic measurements inside a vehicle with different air prototype diffusers, Romanian Journal of Acoustics and Vibration, Volume XIV (2017) 